\title{
SMA imaging of the masers in MWC349A
}

\author{
Jonathan Weintroub ${ }^{1} \dagger$, James Moran ${ }^{1}$, Ramprasad Rao ${ }^{2}$, \\ Hiroko Shinnaga $^{3}$, David Wilner ${ }^{1}$ and Ken Young ${ }^{1}$ \\ ${ }^{1}$ Harvard-Smithsonian CfA, 60 Garden Street, MS78, Cambridge, MA 02138, USA \\ email: jweintroub@cfa.harvard.edu \\ ${ }^{2}$ ASIAA, 645 North A'ohoku Place, Hilo, Hawaii 96720 \\ ${ }^{3}$ Caltech Submillimeter Observatory, 111 Nowelo Street, Hilo, HI 96720
}

\begin{abstract}
In September 2004 and September 2005 we used the Submillimeter Array (SMA) to map the distribution of hydrogen recombination line (HRL) maser spots that originate in the envelope of the peculiar star MWC349A. We measured positions in the line over a $100 \mathrm{~km} / \mathrm{s}$ velocity range with $1 \mathrm{~km} / \mathrm{s}$ spectral resolution. Relative angular position was estimated to an accuracy of about 0.01 arcseconds. The $\mathrm{H} 30 \alpha$ maser components can be tracked continuously in frequency between the red- and blue-shifted peaks of the spectrum. The masers appear to trace a nearly edge-on disk-like structure. The position-velocity relationship is quite remarkable, and reminiscent of that of NCG4258. However, the "wings" in the PV relation are too steep for a simple Keplerian prescription to be correct. We present our data, and suggest a simple model to account for the steep wings.
\end{abstract}

Keywords. masers, instrumentation: high angular resolution, submillimeter, radio lines: stars

\section{Introduction}

The hydrogen recombination line emission from the peculiar B[e] star MWC349A is double-peaked at frequencies above $100 \mathrm{GHz}$, and is thought to be due to masering in a gaseous rotating circumstellar disk. The star also has an ionized stellar wind outflow. The outflow is suggested by VLA continuum maps [for example Tafoya et al. (2004)] showing an hourglass structure, as well as a characteristic spectral index of 0.6. Maser spot maps from OVRO [Planesas et al. (1992)], show two spots, one associated with each spectral peak, centered about 0.05 arcseconds apart with a position angle of about $107^{\circ}$. The position angle of the spots is aligned with that of the waist of the hourglass structure in the continuum map, suggesting that the maser spots are located in the waist. Figure 1 shows the VLA continuum data from White and Becker (1985) with the Keck aperture masking data of Danchi et al. (2001) and the OVRO $230 \mathrm{GHz}$ spotmap of Planesas et al. (1992) overlaid.

\section{Results}

In this paper we present SMA images of the maser emission in the $\mathrm{H} 30 \alpha$ line which show much more detail than the OVRO map. The upper panel of figure 2 shows the maser spot positions derived from the SMA data plotted as a spotmap. The position of the two spot clusters is consistent with the OVRO map in that they are centered approximately $0^{\prime \prime} .05$ apart, however there are many more spots. Spots are also present between the clusters in an elongated distribution. The lower panel shows radial velocity against RA.

$\dagger$ Present address: SAO, 60 Garden Street, MS-78, Cambridge, MA 02138 


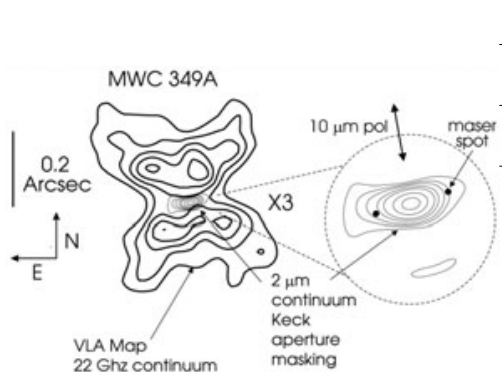

Figure 1. Taken from Danchi et al. (2001), this figure shows an overlay of representative continuum data and $\mathrm{H} 30 \alpha$ interferometric data published on MWC349A prior to this work.
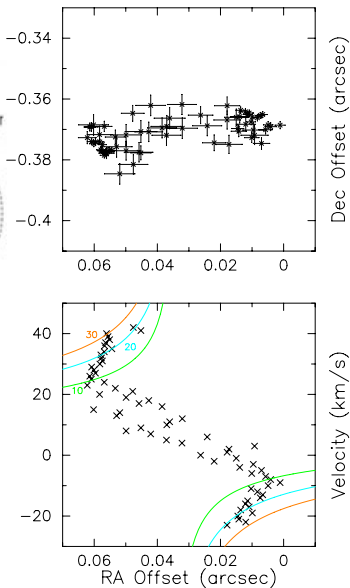

Figure 2. The SMA spotmaps and a PV diagram.

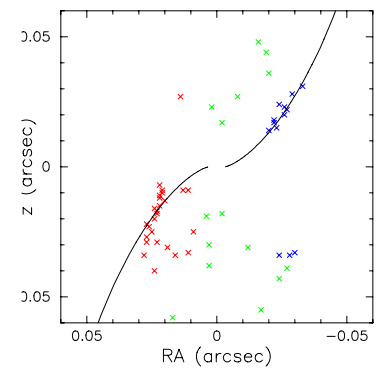

Figure 3. A deprojected SMA $\mathrm{H} 30 \alpha$ spotmap made under the assumptions discussed in the text, with a possible spiral model for the maser spot distribution overlaid

This PV diagram shows a region of apparent solid body rotation with wings reminiscent of the Keplerian wings of NGC4258. However as the three overplotted Keplerian curves $\left(10,20\right.$ and $\left.30 \mathrm{M}_{\odot}\right)$ show, the wings in the measured data are in fact much steeper. An alternate explanation for the apparent solid body region is that the masers between the clusters might be concentrated in an annular ring.

Figure 3 shows a deprojected view of the maser distribution along an axis perpendicular to the line of sight and the axis of the maser distribution. The azimuth angle is determined by assuming Keplerian rotation. The red- and blue-shifted features are assumed to arise in front of and behind the midline, respectively. The rapid change in azimuth angle with velocity can explain the steepness of the PV plot, suggesting a spiral distribution of maser spots; a possible spiral model is overplotted.

\section{Conclusions}

With more baselines, greater sensitivity and a superior site, the SMA has allowed us to closely examine the dynamics of the stellar disk in MWC349A. We show that either the circumstellar disk is not in simple Keplerian rotation, or, if the disk rotation is Keplerian, we suggest that the high velocity masers may trace spiral density arms. We are working on more elaborate dynamical models which combine the disk rotation with the outflow.

\section{Acknowledgements}

The Submillimeter Array is a joint project between the Smithsonian Astrophysical Observatory and the Academia Sinica Institute of Astronomy and Astrophysics and is funded by the Smithsonian Institution and the Academia Sinica.

\section{References}

Danchi, W. C., Tuthill, P. G., \& Monnier, J. D. 2001, ApJ, 562, 440

Planesas, P., Martín-Pintado, J., \& Serabyn, E. 1992, ApJ, 386, L23

Tafoya, D., Gómez, Y., \& Rodríguez, L. F. 2004, ApJ, 610, 827

White, R. L. \& Becker, P. H. 1985, ApJ, 297, 677 